\title{
A cooperative OA system based on SaaS supporting multi-tenant business process customization
}

\author{
Hua Huang \\ Yi-lai Zhang \\ Jiangxi Ceramic Enterprise Information Jiangxi Ceramic Enterprise Information \\ Technology Engineering Research Center,Technology Engineering Research Center, \\ Jingdezhen Ceramic Institute Jingdezhen, Jingdezhen Ceramic Institute Jingdezhen, \\ Jiangxi \\ 13970398420, China, 3330011 \\ jdz_hh@qq.com \\ Jiangxi \\ 13707989962,China, 3330011 \\ jdzzyl@qq.com
}

\author{
Zaiwen Feng \\ State Key Laboratory of Software \\ Engineering, School of Computer, \\ Wuhan University \\ 13554119477,China,430072 \\ 191148074@qq.com
}

\begin{abstract}
The cooperative OA system is a subsystem of the ceramic cloud technology service integration platform, which is a multi-tenant application software based on SaaS. With the use of database accessing middleware and the technology of single sign-on (SSO) and role-based rights management, it integrates and shares the resources of other subsystems in this platform. A framework for multi-tenant business process customization is pulled into this system, through the component of business process visualization self-definition; this system implements multi-tenant business process customization for meeting individual business requirements of different company tenant's online office.
\end{abstract}

Categories and Subject Descriptors

H.4.2 [Information Systems Applications]: Types of Systems---OA

General Terms: Design and Implement.

Keywords-SaaS;Multi-tenant management; Business process customization; cooperative $\mathrm{OA}$.

\section{INTRODUCTION}

At present a network office system is developed for an enterprise according its actual requirements, then deployed within the enterprise's LAN, which leads to many problems, such as too expensive software and hardware cost for running these OA systems, too large maintenance cost for the second development when new requirements come[1]. Progress of office automation for SMEs (Small and Middle Enterprises) is often hampered by these traditional OA.

The SaaS (Software as a Service) promotes the concept of leasing mode, focusing on the software which provides more feature-rich applications for SMEs but with lower cost, the tenant can achieve the requirements of enterprise network office only by a browser. Therefore, building a cooperative OA system based on SaaS supporting multitenant business process customization will provide a great convenience for the realization of office automation of the SMEs.

This cooperative office system is a subsystem of the ceramic cloud technology service integration platform, which is a cloud service platform based on SaaS model, mainly provides business management services, such as OA / CRM / ERP / DSS , etc., design services, such as

This work was supported by National Science and Technology Support Program (NO.2012BAH25F02 and No. 2013BAF02B01) ceramic pattern design/ceramic formulation design, transaction services, such as product or order transaction, advisory services, etc, As long as enterprises tenants have registered and pay a fee, the cooperative office system in the platform will be available to them.

\section{RELATED WORK}

There are currently some successful cases of SaaS application, such as Salesforce, the founder of the SaaS model, which is one of the most successful companies applying SaaS mode, and has developed online CRM application software, that is a great success. Google is also a pioneer in SaaS industry, which has developed a large number of online office suites, such as email, word processing, spreadsheets, etc., and Google App Engine was released in 2008, which provides an integrated development environment to run online software for thirdparty developers. Some domestic companies, such as the 800APP company, seize the characteristics of Chinese users, and focus on the development and operation of online customer relationship management system (CRM). In addition, the product line of the 800APP has been extended to OA. The traditional software of Kingdee have be transformed into SaaS mode and moved to the Internet, taking the first step in the development of SaaS strategy [2]. Although there are already some successful cases, but most applications are only just beginnings in the entire SaaS industry.

The SaaS has three layer: presentation layer, interface layer and application layer [3], An important feature of which is the single instance and multi-tenant, that multiple tenants share an application example provided by service providers [5] .In order to meet the different tenants' personalized business process requirements, The application layer of SaaS must be emphasized to achieve the capabilities of configuration and resource sharing, making a SaaS application can serve multiple tenants well [3].Creating a SaaS application owning to the tenant on the basis of shared instance is necessary, there are many SaaS application configuration problems studied by some scholars[4][10]. The three layers of process, service and data are mainly related to the SaaS customization. The research content of collaborative office system studied by 
this paper mainly involves multi-tenant business process customization.

As to the research of business process customization there are a lot of works around the dynamic binding, service optimization, configurable process model [5-9], while the research of business process customization of the SaaS mode focuses on the configurable process model. In[4],Zhao promotes a configurable process model process for SaaS, in which the services and configurable rules, association and constraint functions are clearly defined, so that the services can be separated from the business logic, but the model lacks the support for generating multi-tenant instances and the complex process configuration. In [10] Mietzner puts forward a process model, which can be customized based on the description of change for SaaS applications, the model is personalized from a given template according the tenant's needs, and then be converted into a BPEL process model, the BPEL Language is extended into VxBPEL language in the paper [11], allowing business processes to be customized based on the changing points, which are needed to be determined through some parameters at runtime.

In summary, the business process modeling and customization for SaaS is also still in its infancy, although there are some studies, but the established process model is not perfect, which is either too complex, or difficult to be achieved. So we propose a simple and practical framework of multi-tenant business process customization, and give the key technology and service components for realizing collaborative office systems based on SaaS: single sign-on (SSO) technology, role-based rights management technology, database accessing middleware, visual business process definition component.

\section{SYSTEM FUNCTION DESIGN}

The functions of this cooperative office system based on SaaS can be divided into two parts: basic service module and OA module. Basic service module is to manage the entire system, mainly for software service provider. OA module is used primarily for enterprise tenant.

\subsection{Function design of Basic Service Module}

The basic service module is the common service module to provide support for the development of other business function modules. In the SaaS mode, we design the specific process of using services: SaaS providers publish service system and provide selection and entrance of lease agreements for service system, after the tenants have registered successfully, they can configure enterprise information, organizational structure and personnel, using role-based authorization to manage users' rights. The main functional modules of this platform are shown in Figure 1.

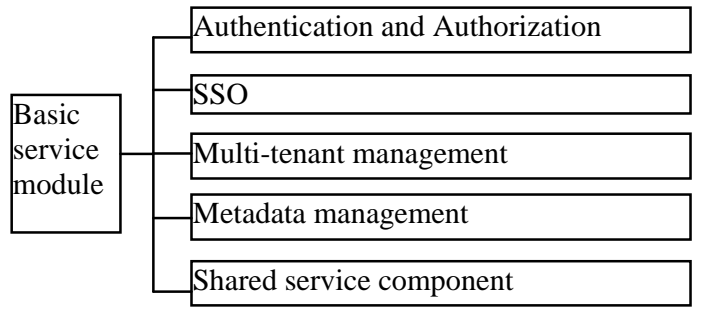

Figure 1. Basic service platform functional diagram

The function of each sub module in the basic service module is described as follows:

(1)Metadata Management: This module is a configurable functional module; the enterprise tenants choose the different services and interface style to configure basic data of enterprises according to their requirements.

(2)Multi-tenant management: This module is mainly responsible for managing tenants' basic information

(3)Authentication and authorization: This module is to ensure the safety of all tenants using system and to protect tenants' data from infringement.

(4)Shared service component: This module is mainly to separate common function from the unrelated class; Major public components and services include: database accessing middleware, cache management component, log management component, as well as some other core runtime component services.

(5) Single sign-on (SSO): Tenants and their internal users can access to other subsystems without re-login after having login in one system. As a highly scalable SaaS platform, SSO is a prerequisite for scalable service; Secondly with the infinite tenants' requirements, the platform should be able to integrate other software systems, so this module must be implemented in this platform.

\subsection{Function design of $\mathrm{OA}$}

The main functions of this cooperative office system are showed as below:

(1)Handle personal office affairs, create a personal document management, e-mail, and address book, daily schedules, and work management;

(2)Establish an information sharing mechanism for the registered enterprises, branch offices and departments, including announcements, internal news, community forums, events, community forum, public information and document archival information sharing and retrieval;

(3)Daily administration of receipt and dispatch enterprise management, make dispatching, receiving messages and circulating documents to be an automatic paperless circulation;

(4)Support enterprise tenants to customize business processes;

(5)Achieve the integration of office systems and financial management, internal business data processing system. 


\section{THE FRAMEWORK SUPPORTING MULTI-TENANT BUSINESS PROCESS CUSTOMIZATION}

\subsection{Framework Design}

This paper presents a simple framework supporting multi-tenant business process customization to achieve online office workflow customization for tenants. The architecture of this framework is shown in Figure 2, which consists mainly of four parts: Visual Process Definer, Business Object Table, Process Engine, and Tenant Process Database.

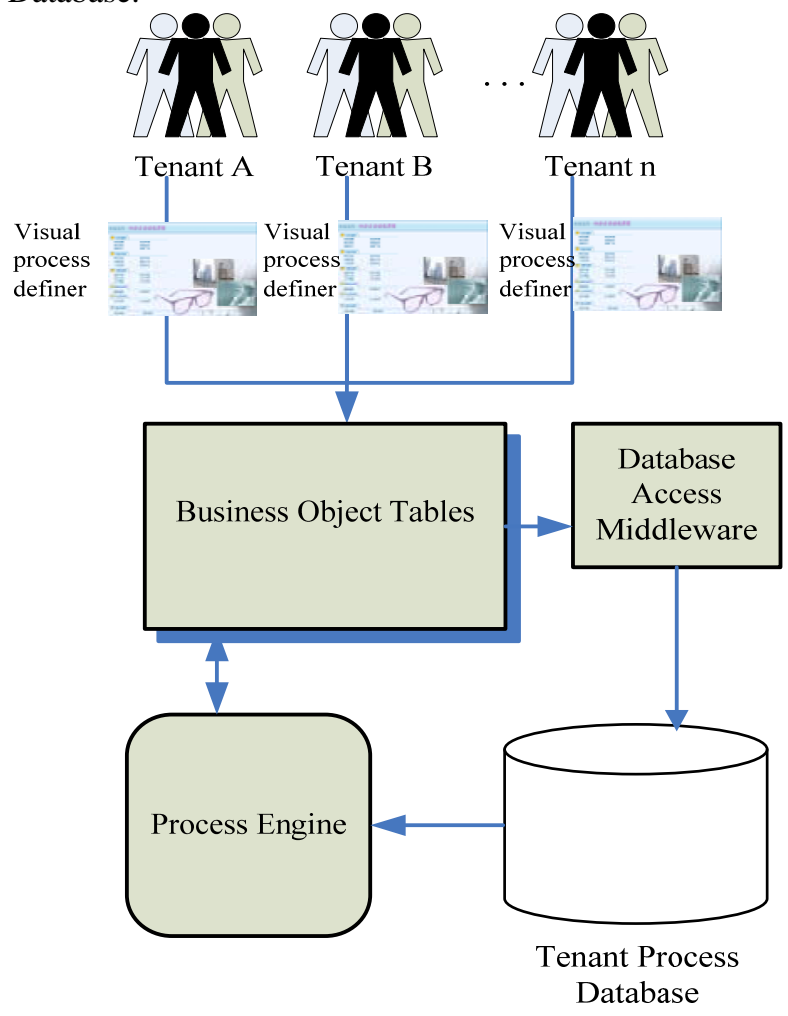

Figure 2. The framework diagram of multi-tenant business process customization

(1)Visual Process Definer: it is a web page for visual definition and editing of tenant' process. A number of business process conditions defined by the description of natural language, actions templates and some related business objects and their properties are provided in the definer in advance, tenants can easily design business processes in line with their requirements by the definer .

(2)Business Object Table: Each tenant has a business object table to store business objects related to business processes defined by tenants.

(3)Process Engine: Being used for accepting data input, interpreting, executing business processes.

(4)Tenant Process Database: Through the database accessing middleware to storage process definition information of each tenant and related-processes application data according to tenant ID.
In this framework, the reason to set up a business object table for each tenant is that meeting each tenant's individual business requirements leads to the number of the business logic defined by all tenants may be very large. A lot of business logic may be referred to a large number of business objects, which also means that a lot of calling points will be pre-defined in process engine for SaaS application. For one tenant, it probably just needs a small part; a lot of unnecessary process engine calling may significantly reduce the execution performance of the system. Therefore,we have established business object table for each tenant, when tenants are defining business processes, the related business objects will be added to the business object table; Before SaaS applications call process engine, they will first search tenants' business object table, if the business object involved in this calling point exists in the tenant's business object table, the process engine is called, otherwise the process engine is not called. The performance of this system is well optimized through such methods.

\subsection{Procedure of Multi-tenant Business Process} Customization

The procedure of business process customization based on multi-tenant business process customization framework under this system is showed as follows:

(1)Business Process Definition: In the interface of process design of the visualization process definer, a tenant can select process nodes, flow control lines, and then fill node's information, such as function description, execution conditions, participating roles, etc, then to combine control line, finally form a complete business process. At the same time, the system will automatically add business objects related to defined tenant business processes to the tenant's business object table.

(2)Business Process Storage: In order to query and modify tenant business processes, the process ID, name, node attributes, execution conditions, node action of tenant business processes are stored in tenant's process database according to tenant ID through the database accessing middleware. If a process of the tenant database has been modified, the related business object table must be modified.

(3)Business Process Execution: When a tenant has log into the system, the system will load the tenant's relatedprocess data into the tenant's process engine rule base used for the process engine. When the system is running to the calling point of the process rule engine, the system will first check the tenant's business object table to determine whether the process rule engine will be called. If the business objects related to the current calling point exists in the current tenant's business object table, the system will call the process rule engine to perform the business logic defined by tenant; otherwise it will not be called 


\section{KEY TECHNOLOGY AND SERVICE COMPONETS OF IMPLEMENTING SYSTEM}

This coordination office system is implemented under the developing framework of ceramic cloud technology service integration platform, the main technology and application service components include: single sign-on (SSO) technology, role-based rights management technology, database accessing middleware, visual business process definition component. The details of them are presented as bellow.

\subsection{Single Sign-On (SSO)}

SSO refers to access a variety of different resources only by one authentication. This authentication method not only increases the security of the system, but also improves the efficiency of employee's work. Users access the unified login page of the ceramic cloud technology service integration platform, as shown in Figure.3,the user's identity is verified by authentication server, the user can have a Cookie (or Session) with a token, its identity information is included in this token, hereafter the user accesses other resources of the platform, the policy agent deployed in the application resources side will confirm his identity based on the token, eliminating the trouble for users to re-enter authentication information.

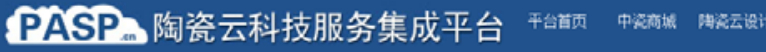

\section{登录 Login}

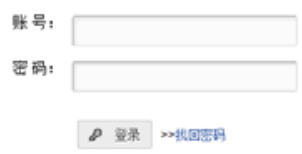

Figure 3. Unified login interface of this platform

The application systems of this platform are usually distributed in their respective IDC, users will undoubtedly involve the cross-domain access of different applications of this platform, and once crossing different domains, the token held by users and stored in Cookie (or Session) will disappear, SSO will not be completed. To solve the crossdomain problem of SSO, a CDC (Cross Domain Controller) can be built on the authentication server of this platform, and some CD SSO Servlets will be established on each application server, so the cross-domain problem of SSO can be resolved through the communication between CDC and CD SSO Servlet.

\subsection{Role-based rights management technology}

Role-based rights management model is proposed by the American National Standards and Technical Committee (NIST) in the 1990s. The model successfully introduced the role of intermediary, the application systems define various roles according to tenants' requirements, and set appropriate accessing rights, the company tenants can assign different roles to internal staffs according to their office duties and tasks. The entire process of rights management is divided into two parts: association between accessing rights and roles, association between roles and users, which achieve a logical separation between users and their accessing rights. The relationship is shown in Figure 4.

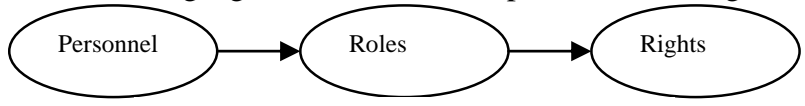

Figure 4 RABC simplified diagram

In the procedure of tenant business process customization, for process participants, we apply the role associated with a specific tenant's internal staff, for personnel changing; it is only necessary to modify the association relation between roles and personnel, unnecessary to alter the definition information of the tenant's business processes, so the flexibility and adaptability of customizing processes is enhanced.

The advantages of role-based rights management that can expressed the complex relationship between internal organization and personnel by roles, which can be departments or job's positions, it is flexible to switch between different roles for personnel, which makes it easy to manage licenses and activities participants of tenant business process. The interface of assigning roles and permissions for each tenant personnel in this system is shown in Figure. 5

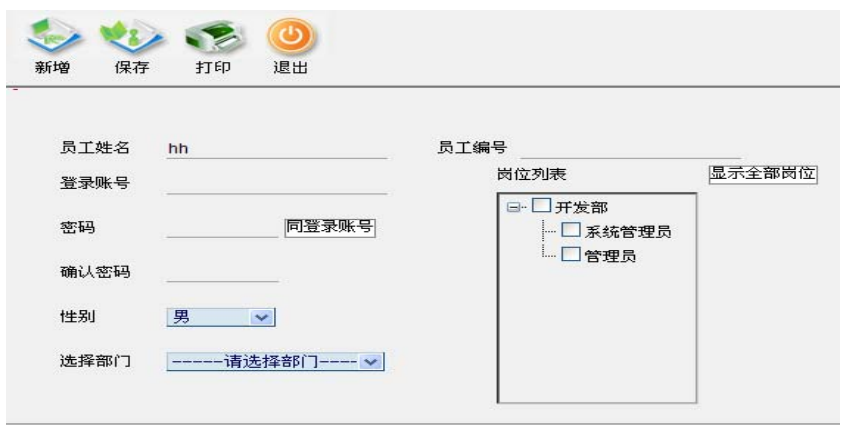

Figure 5.The interface of assigning roles and rights for each tenant personnel

\subsection{Database accessing middleware}

In order to ensure the consistency of various subsystems accessing database under the ceramic cloud technology service integration platform , and to address the interoperable mode of data application resources built in their corporate databases for multi-tenant, to support that process-related information and data stored in tenants process database can be accessed and operated according to tenant ID during multi-tenant business processes customization, a database accessing middleware for SaaS mode is designed, which is composed of two components: multi-enterprise database connecting component (Getcnstr) and data manipulating components (SqlData). The latter 
mainly provides two overloaded public functions used for manipulating data: RunSql, RunProcedure. The work principle of database middleware in this platform is as shown in Figure.6

1.8 Implementing visual self-defining components of multi-tenant business process

In order to implement visual self-defining components of multi-tenant business process, we describe a business process by the following four elements. (1)Process Participant: defining executor of activities, who can be person or other entities. (2) Process Activity: defining the activity steps of a business process, which can be divided into atomic activity and sub-process, the former cannot be divided, the latter can be composed of atomic activities and other sub-process.(3)Process Relevant Data: defining the data information involved in process activities and applications, which may contain the data of system and environment.(4)Transition Information: defining related information for carrying out business process activities, determining the transition between activities and the impact on the implementation of other activities when completing an activity

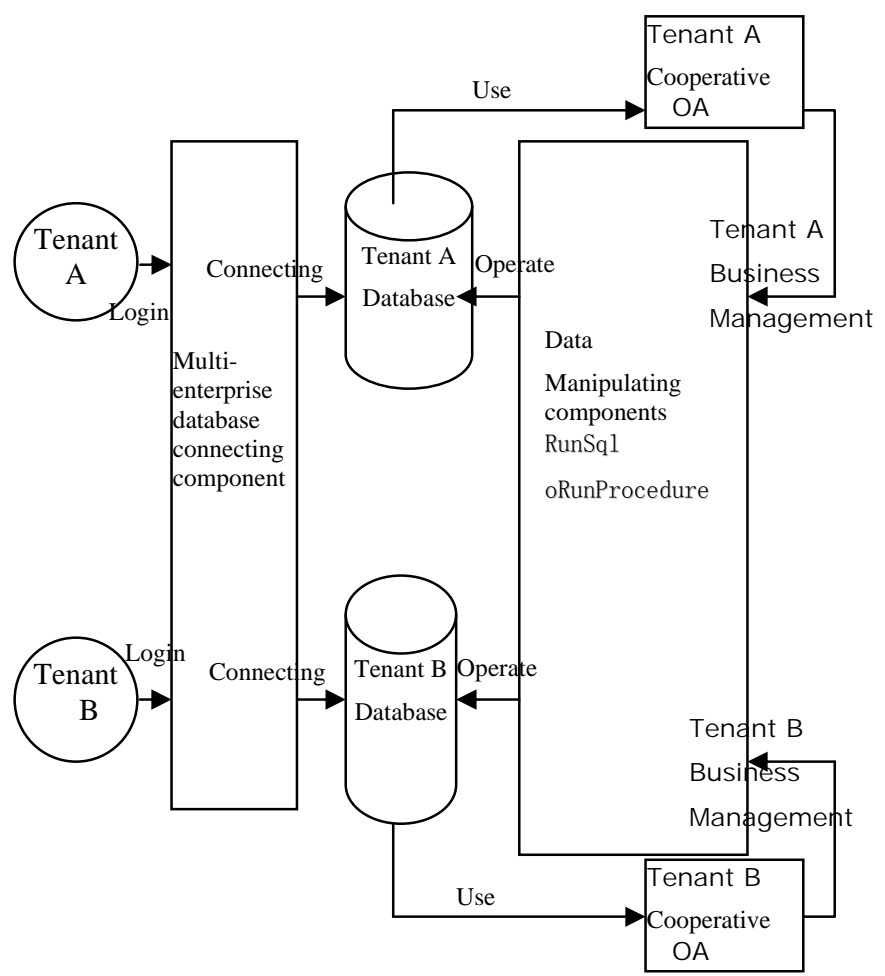

Figure 6.The work principle diagram of database middleware
The basic elements above involved in the process are described by the vector graphics SVG in Figure 7. The Start node and the End node is represented by the ellipse, a rectangle represents Processing node, The Branch node is

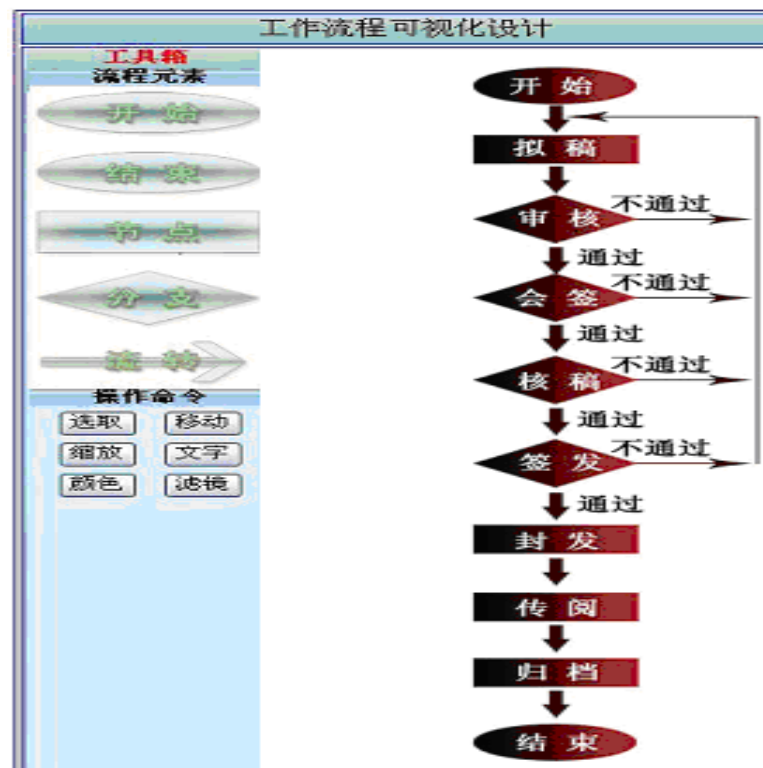

Figure 7. Interface of business process visualization self-definition

represented by diamond, The personnel, the name of performing step or operation and other relevant data are dynamically set in the pop-up dialog of each node, process flow and trigger conditions are indicated by arrows, each process is executed from the Start node to the End node.

When designing a process, the tenant clicks the button in the toolbox, and then drags the visual component onto the working place; each process visualization component represents a node, which is associated with other components by the flow arrows. During the operations, and the trigger conditions and flow direction of the process are set by its property dialog. Visual definition of tenant's business process is completed, then to click the Save button in the menu area, the information related to the defined business process is saved to the tenant process database according to tenant ID.

\section{CONCLUSIONS AND OUTLOOK}

The cooperative office system is multi-tenant application based on the SaaS mode; it integrates and shares the resources and information of other subsystem of the ceramic cloud technology service integration platform by the technologies of database accessing middleware, single sign-on and role-based rights management. This system achieves tenant business process customization to meet the individual needs of each tenant online office by applying the framework of multi-tenant business process customization. During the process of developing system, there are some works needed to be improved in future. On the one hand, due to the constraints of time, technology and 
other aspects, multi-tenant intelligent workflow engine in this paper is not promoted, which considers the executing efficiency of multi-tenant business processes. On the other hand, the customization of multi-tenant business process is achieved not by the process customization model of the SaaS mode, but through a tenant private process database and business object table based on the model of traditional workflow, which is a simple and practical way to achieve multi-tenant business process customization, so this system does not support the complex customization of large enterprise business processes. In view of this, to build a business process customization model for SaaS mode and through which to improve the efficiency of multi-tenant business processes and the applicability of this system is the focus of future research.

\section{REFERENCES}

[1] Xie Yi-ming. SaaS is achieved by integration of Internet and software [J]. Software world,2006.15:31.

[2] Tang Tiebing. Research and implementation of enterprise job scheduling system based on SaaS[D]. Computer software and theory of Chongqing University, 2008.

[3] Zhao Li-jun. Development and evolution of SaaS technology[J]. Modern telecommunications technology, 2007,37 (12) :46-48.

[4] Shi Yu-liang,Luan shuai, LI Qing-zhong. Research of SaaS business process customization and verification mechanism based on TLA[J]. Journal of Computers, 2010,11.
[5] Liu Shu-lei,Liu Yun-xiang. Global optimal dynamic service selection algorithm of QoS-aware service composition[J] Journal of Software, 2007,18 (3): 646656.

[6] Jaeger,M.C., Muhl,G., Golze,S. QoS-aware composition of Web services:A look at selection algorithm[C].Proceedings of the 2005 IEEE International Conference on Web Services (ICWS).Or-lando,FL,USA,2005: 807- 808.

[7] Menasc'e,D.A,Dubey V.Utility based QoS brokering in serviceoriented architectures[C].Proceedings of the 2007 IEEE International Conference on Web Services ( ICWS).Salt Lake City,Utah,USA,2007: 422-430.

[8] Alrifai,M.,Risse,T. Combining global optimization with local selection for efficient QoS-aware service composition[C]. Proceedings of the 18th International World Wide Web Conference(WWW). Madrid, Spain, 2009: 881-890.

[9] Mietzner,R.,Leymann,F.Generation of BPEL Customization Processes for SaaS Applications from Variability Descriptors. In: Services Computing (2008SCC), pp. 359-366.

[10] Michiel, K., Chang-ai, S., Marco, S., Paris, A.: VxBPEL: Supporting variability for Web services in BPEL. Information and Software Technology 51, 258-269 (2009). 